\title{
Three-Dimensional Nanostructure and Volume Fraction Measurements of Porous Titania Photocatalysts by Electron Tomography and Their Relation to Photo-Catalytic Activity
}

\author{
K. Yoshida, ${ }^{*}$ N. Tanaka, ${ }^{* *}$ E.D. Boyes, ${ }^{* * *}$ and P.L. Gai****
}

* Nanostructures Research Laboratory, Japan Fine Ceramics Center, 2-4-1 Mutsuno, Atsuta-ku, Nagoya, 456-8587, Japan

** Department of Crystalline Materials Science and Ecotopia Science Institute, Nagoya University, Furo-cho,Chikusa-ku, Nagoya, 464-8603, Japan

*** Departments of Physics and Electronics, The York JEOL Nanocentre, The University of York, Heslington, York TO10 5BR,UK

**** Departments of Chemistry and Physics, The York JEOL Nanocentre, The University of York, Heslington, York TO10 5BR,UK

Since the discovery of the Honda-Fujishima effect in 1972, many researchers have been developing methods to achieve high photocatalytic activity of titania by controlling its structure on the nanoscale. Such methods include, the production of fine particles in highly porous configurations with large surface areas, formation of metal clusters adhered to the particle surfaces to act as effective reaction sites [1], and nitriding of the $\mathrm{TiO}_{2}$ surface for visible light activation [2]. However, it has been rather difficult to analyze these kinds of complex heterogeneous structures in titania photocatalysts using conventional spectroscopic or diffraction methods.

Transmission electron microscopy (TEM) is one of the most effective methods for analyzing such heterogeneous catalysts, although the images obtained are limited to two dimensions (2D). In 1992, electron tomography was proposed as a means of obtaining three-dimensional (3D) structural information from 2D TEM images [3]. The electron tomography has been applied to the examination of particle catalysts [4]. In electron tomography, 3D structures of samples are reconstructed using many TEM images obtained from various directions. The reconstruction algorithm is the same as that used in computed tomography (CT) by X-rays. In recent years, studies have shown that electron tomography using high angle annular dark field scanning transmission electron microscopy (HAADF-STEM) has become an even more powerful method for observing 3D structures of crystalline materials compared with TEM images. In the present study we have firstly applied the tomographic method based on STEM to analyze 3D nanostructures of photocatalytic titania.

Various porous titania photocatalysts were analyzed three dimensionally in real space by electron tomography. Shapes and 3D distributions of fine pores and silver (Ag) particles (2 nm in diameter) within the pores were successfully reconstructed from the 3D data (FIG.1). Electron tomography was more accurate for measuring the specific surface area than the general BET method, when the internal voids in the sample are closed. Calculated specific surface areas of $22.8 \mathrm{~m}^{2} / \mathrm{g}$ for a conventional sol-gel $\mathrm{TiO}_{2}$ sample and $366 \mathrm{~m}^{2} / \mathrm{g}$ for a highly porous $\mathrm{TiO}_{2}$ sample prepared using the Pluronic P-123 self-assembly process indicate that the highly porous $\mathrm{TiO}_{2}$ produced by the present method using block copolymers has a greater number of effective reaction sites for the degradation of methylene blue. Quantitative measurement of silver particles of the modified porous $\mathrm{TiO}_{2}$ sample clarified a diffusion constant of Ag cations in nanopores (FIG.2). The results showed that electron 
tomography contribute significantly to the nanostructural analysis and design of such catalyst materials for photocatalysis.

\section{References}

[1] M. Haruta, Catalysis Today 36 (1997) 15.

[2] R. Asahi et al., Science 293 (2001) 269.

[3] J. Frank, Electron Tomography: Three Dimensional Imaging with the Transmission Electron Microscope, Plenum, New York, London, 1992.

[4] P. A. Midgley et al., Ultramicroscopy 96 (2003) 413.

[5] This study was partly supported by a Grant of JSPS Research Fellowship for Young Scientists (K. Y.). We thank Mr. S. Sueda for assistance with the image processing. We thank the University of York, regional development agency, Yorkshire Forward, the European Union through the European Regional Development Fund and JEOL (UK) Ltd for the Nanocentre facilities.

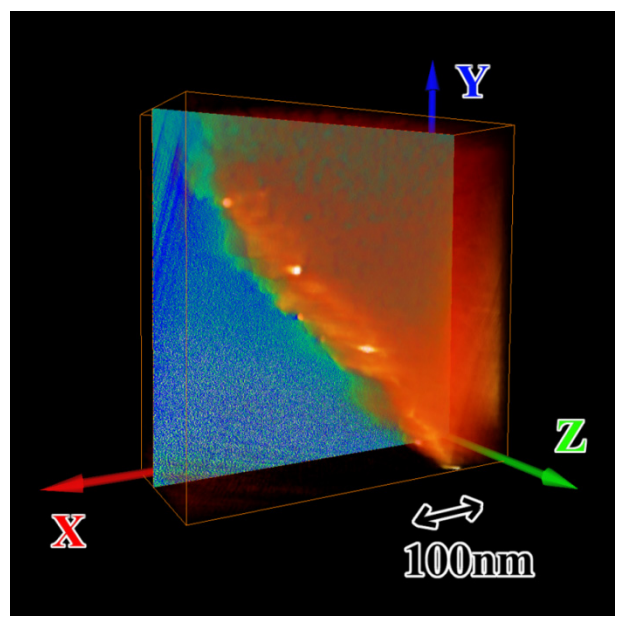

FIG. 1. Reconstructed 3D structure of the conventional $\mathrm{TiO}_{2}$. The green, red and blue arrows show the incident axis of the electron beam ( $\mathrm{Z}$ axis), the rotation axis of tomography ( $\mathrm{Y}$ axis) and another $\mathrm{X}$ axis, respectively. The scale bar is an estimate only. The voltex image was also produced $\left(\right.$ amira ${ }^{\circledR}$, Visage

FIG. 2. TEM/STEM images of the $\mathrm{Ag} / \mathrm{TiO}_{2}$ using double Cs corrected thermal fieldemission HRTEM (JEOL: JEM-2200FS). (a) shows low-magnification Cs-correction STEM image. (b) is high-resolution Cscorrection STEM image (b), corresponding $4.5 \mathrm{~nm}$ under-focused Cs-correction TEM image (c) and $4.5 \mathrm{~nm}$ over-focused Cscorrection TEM image (d).

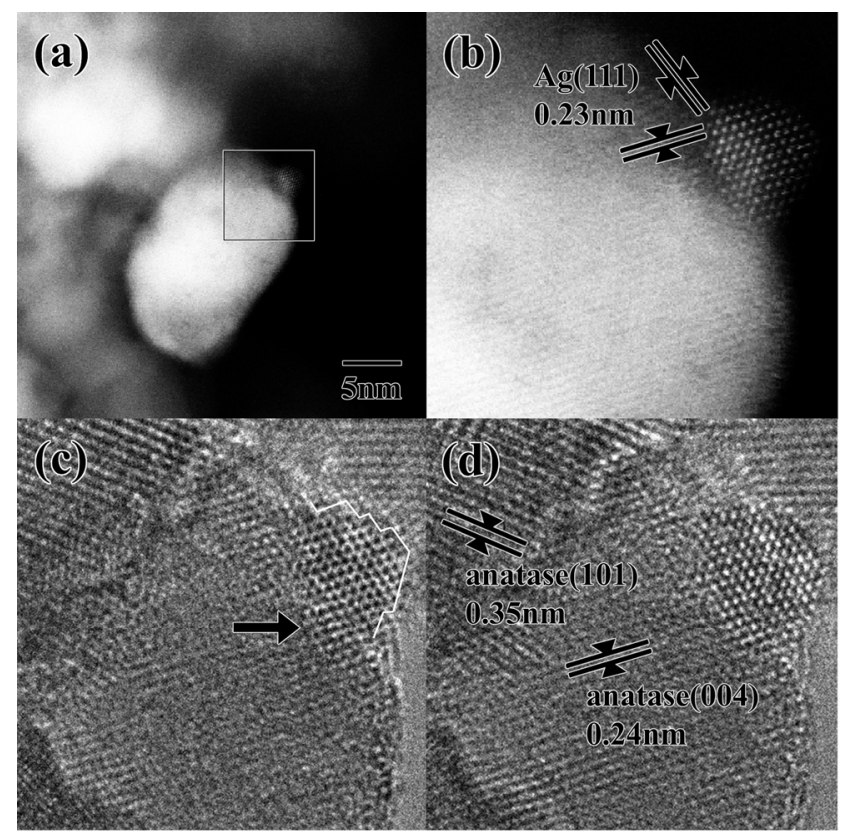

TABLE 1. 3D morphology of the conventional $\mathrm{TiO}_{2}$ and the superporous $\mathrm{TiO}_{2}$.

\begin{tabular}{lclll}
\hline \multicolumn{2}{c}{ Void Volume Fraction [\%] } & \multicolumn{1}{c}{ Surface Area $\left[\mathrm{nm}^{2}\right]$} & $\mathrm{SSA}_{\text {tomo }}\left[\mathrm{m}^{2} / \mathrm{g}\right]$ & $\mathrm{SSA}_{\mathrm{BET}}\left[\mathrm{m}^{2} / \mathrm{g}\right]$ \\
\hline Conventional $\mathrm{TiO}_{2}$ & 9.4 & $8.36 \times 10^{5}$ & 79.1 & 22.5 \\
Superporous $\mathrm{TiO}_{2}$ & 41.2 & $2.64 \times 10^{6}$ & 383 & 349 \\
\hline
\end{tabular}

